\title{
$1 \quad$ Automated skull stripping in mouse fMRI analysis using 3D U-Net
}

2 Guohui Ruan ${ }^{1,2}$, Jiaming Liu ${ }^{1,2}$, Ziqi An ${ }^{1,2}$, Kaiibin $\mathrm{Wu}^{1,2}$, Chuanjun Tong ${ }^{1,2}$, Qiang $\mathrm{Liu}^{1,2}$,

3 Ping Liang ${ }^{3}$, Zhifeng Liang ${ }^{4,5}$, Wufan Chen ${ }^{1,2,6,7}$, Xinyuan Zhang ${ }^{1,2,6,7, *}$, Yanqiu Feng ${ }^{1,2,6,7, *}$

4 1. School of Biomedical Engineering, Southern Medical University, Guangzhou, China;

5 2. Guangdong Provincial Key Laboratory of Medical Image Processing, Southern Medical

6 University, Guangzhou, China;

7 3. XGY Medical Equipment Co. Ltd., Ningbo 315400, China ;

8 4. Institute of Neuroscience, CAS Center for Excellence in Brain Sciences and Intelligence

9 Technology, Key Laboratory of Primate Neurobiology, Chinese Academy of Sciences, Shanghai 200031, China;

5. Shanghai Center for Brain Science and Brain-Inspired Intelligence Technology, Shanghai 201210, China;

6. Guangdong Province Engineering Laboratory for Medical Imaging and Diagnostic 14 Technology, Southern Medical University, Guangzhou, China;

7. Guangdong-Hong Kong-Macao Greater Bay Area Center for Brain Science and Brain-Inspire d Intelligence \& Key Laboratory of Mental Health of the Ministry of Education, Southern Me dical University, Guangzhou, China

* Corresponding author at: School of Biomedical Engineering, Southern Medical University, 


\section{Abstract}

24 Skull stripping is an initial and critical step in the pipeline of mouse fMRI analysis.

25 Manual labeling of the brain usually suffers from intra- and inter-rater variability

26 and is highly time-consuming. Hence, an automatic and efficient skull-stripping

27 method is in high demand for mouse fMRI studies. In this study, we investigated a

28 3D U-Net based method for automatic brain extraction in mouse fMRI studies. Two

29 U-Net models were separately trained on T2-weighted anatomical images and

$30 \mathrm{~T} 2 *$-weighted functional images. The trained models were tested on both interior

31 and exterior datasets. The 3D U-Net models yielded a higher accuracy in brain

32 extraction from both T2-weighted images (Dice $>0.984$, Jaccard index $>0.968$ and

33 Hausdorff distance $<7.7$ ) and T2*-weighted images (Dice $>0.964$, Jaccard index >

340.931 and Hausdorff distance < 3.3), compared with the two widely used mouse

35 skull-stripping methods (RATS and SHERM). The resting-state fMRI results using

36 automatic segmentation with the 3D U-Net models are identical to those obtained by

37 manual segmentation for both the seed-based and group independent component

38 analysis. These results demonstrate that the $3 \mathrm{D}$ U-Net based method can replace

39 manual brain extraction in mouse fMRI analysis.

40

41 Keywords: Skull stripping, Deep learning, 3D U-Net, Mouse, fMRI

42 
43

44

45

46

47

48

49

50

51

52

53

54

55

56

57

58

\section{Introduction}

Functional magnetic resonance imaging (fMRI) (D'Esposito et al., 1998; Lee et al., 2013) has been widely employed in neuroscience research. The key advantage of mouse fMRI (Jonckers et al., 2011; Mechling et al., 2014; Perez-Cervera et al., 2018;

Wehrl et al., 2014) is that it can be combined with neuromodulation techniques (e.g., optogenetics) and allows manipulation and visualization of whole-brain neural activity in health and disease, which builds an important link between pre-clinical and clinical research (Lake et al., 2020; Lee et al., 2010; Zerbi et al., 2019). In mouse fMRI research, structural and functional images are commonly acquired with T2-weighted $(\mathrm{T} 2 \mathrm{w})$ and $\mathrm{T} 2 *$-weighted $(\mathrm{T} 2 * \mathrm{w})$ scanning, respectively. Generally, functional $\mathrm{T} 2{ }^{*} \mathrm{w}$ images need to be registered to standard space using two spatial transformations, which are obtained by registering functional images to anatomical images and subsequently registering anatomical images to a standard space. To exclude the influence of non-brain tissues on image registration, it is necessary to perform skull stripping on both structural and functional images. In the practice of mouse fMRI analysis, skull stripping is usually performed by manually labeling each MRI volume slice-by-slice, due to the absence of a reliable automatic segmentation method. This manual brain extraction is extremely time-consuming, as a large number of slices need to be processed in the fMRI analysis for each mouse. In addition, manual segmentation suffers from intra- and inter-rater variability. Therefore, a fully automatic, rapid, and robust skull-stripping method for both T2w and $\mathrm{T} 2{ }^{*} \mathrm{w}$ images is highly desirable in mouse fMRI studies. 

developed and widely used, including Brain Extraction Tool (BET) (Smith, 2002), Hybrid Watershed Algorithm (HWA) (Segonne et al., 2004), the Brain Extraction based on nonlocal Segmentation Technique (BEaST) (Eskildsen et al., 2012), and the Locally Linear Representation-based Classification (LLRC) for brain extraction (Huang et al., 2014). However, these methods cannot directly be applied for mouse skull stripping. Compared to human brain MR images, mouse counterparts have relatively lower tissue contrast and a narrower space between the brain and skull, which substantially increases the difficulty of mouse brain segmentation. In addition, the $\mathrm{T} 2{ }_{\mathrm{W}}$ images used for mouse fMRI may suffer from severe distortion and low spatial resolution, making the skull stripping of functional images more challenging

76 than that for structural images.

77 Several methods have been proposed for rodent brain extraction. 3D Pulse-Coupled Neural Network (PCNN)-based skull stripping (Chou et al., 2011) is an unsupervised artificial 3D network approach that iteratively groups adjacent pixels with similar intensity and performs morphological operation to obtain the rodent brain mask. Rodent Brain Extraction Tool (T. Wood, 2013) is adapted from the well-known BET (Smith, 2002) method with an appropriate shape for the rodent.

83 Rapid Automatic Tissue Segmentation (RATS) (Oguz et al., 2014) consists of two 84 stages: grayscale mathematical morphology and LOGISMOS-based graph segmentation (Yin et al., 2010), and it incorporates the prior of rodent brain anatomy 
87 filtering (SHERM) (Liu et al., 2020) is an atlas-based method that relies on the fact

88 that the shape of the rodent brain is highly consistent across individuals. This

89 method adopts morphological operations to extract a set of brain mask candidates

90 that match the shape of the brain template, and then merges them for final skull

91 stripping. One of the common disadvantages of the above methods is that their

92 effectiveness was verified only on anatomical images, and cannot be guaranteed on

93 functional images. Another limitation is that the performance of these methods is

94 severely affected by the brain shape, texture, signal to noise ratio, and contrast of

95 images, and hence they need to be optimized for different MRI images. Therefore, it

96 is necessary to develop an automatic skull-stripping method that is effective and has

97 a stable performance on varying types of MR images.

98 Deep learning has gained popularity in varying image analysis tasks, such as

99 organ or lesion segmentation (Guo et al., 2019; Li et al., 2020; Sun et al., 2019), and

100 disease diagnosis (De Fauw et al., 2018; Suk et al., 2017), owing to its excellent

101 performance. As for skull stripping, Kleesiek et al. (2016) first proposed a 3D

102 convolutional neural network (CNN) method for human brain MR images. Roy et al.

103 (2018) trained a CNN architecture with modified Google Inception (Szegedy et al.,

104 2015) using multiple atlases for both human and rodent brain MR images. With the

105 development of deep learning, more advanced architectures of CNN for semantic

106 segmentation have been proposed. As a popular architecture of deep CNN, U-Net

107 has proved to be very effective in the task of semantic segmentation even with a

108 limited amount of annotated data (Ronneberger et al., 2015). Some U-Net based 
methods for rodent skull stripping have been proposed. Thai et al. (2019) utilized 2D

110 U-Net for mouse skull stripping on diffusion weighted images. Hsu et al. (2020)

111 trained a 2D U-Net based model using both anatomical and functional brain images

112 for mouse and rat skull stripping. De Feo et al. (2021) proposed a multi-task U-Net

113 to accomplish both skull stripping and brain region segmentation simultaneously on

114 mouse anatomical images of the mouse brain. These methods were only evaluated

115 using segmentation accuracy with reference to the ground truth mask. However, the

116 effect of skull-stripping algorithm on the final fMRI results remains unexplored, and

117 whether automatic skull stripping can replace the manual approach in mouse fMRI

118 analysis remains an open question.

119 Here, we investigated the feasibility of using 3D U-Net to extract the mouse brain

120 from $\mathrm{T} 2 \mathrm{w}$ anatomical and $\mathrm{T} 2 *$ functional images for the fMRI analysis. We

121 separately trained the U-Net models on anatomical $\mathrm{T} 2 \mathrm{w}$ and functional $\mathrm{T} 2 * \mathrm{~W}$

122 images, considering that anatomical and functional images are acquired with

123 different sequences and have different contrast, resolution, and artifacts. The

124 performance of trained 3D U-Net models is first quantitatively evaluated using

125 conventional accuracy metrics. In addition, we compared the fMRI results separately

126 obtained using the manual and automatic segmentation masks.

\section{Materials and Methods}

\subsection{Datasets}

130 This study includes two different in-house datasets. Both of these two datasets 
were collected in the fMRI study and were reanalyzed for the purpose of the present study. All animal experiments were approved by local Institutional Animal Care and Use Committee.

The first dataset (D1) was acquired from 84 adult male C57BL/6 mice (25-30 g) on a Bruker 7.0T MRI scanner using cryogenic RF surface coils (Bruker, Germany). Both the anatomical data $(\mathrm{T} 2 \mathrm{w})$ and resting-state fMRI data $(\mathrm{T} 2 * \mathrm{w})$ were acquired for each mouse. The T2w images were acquired using a fast spin echo (TurboRARE) sequence: field of view $(\mathrm{FOV})=16 \times 16 \mathrm{~mm}^{2}$, matrix $=256 \times 256$, in-plane resolution $=0.0625 \times 0.0625 \mathrm{~mm}^{2}$, slice number $=16$, slice thickness $=0.5 \mathrm{~mm}$, RARE factor $=8, T R / T E=2500 \mathrm{~ms} / 35 \mathrm{~ms}$, number of averages $=2$. The resting-state fMRI images were then acquired using a single-shot gradient-echo-planar-imaging sequence with 360 repetitions: FOV $=16 \times 16 \mathrm{~mm}^{2}$, matrix size $=64 \times 64$, in-plane resolution $=0.25 \times 0.25 \mathrm{~mm}^{2}$, slice number $=16$, slice thickness $=0.4 \mathrm{~mm}$, flip angle $=54.7^{\circ}, \mathrm{TR} / \mathrm{TE}=750 \mathrm{~ms} / 15 \mathrm{~ms}$.

The second dataset (D2) was obtained from the previous task-state fMRI research (Chen et al., 2020). A total of 27 adult male C57BL/6 mice (18-30 g) were used in this study (Part 1: 13 for auditory stimulation; Part 2: 14 for somatosensory stimulation). The anatomical $\mathrm{T} 2 \mathrm{w}$ images and two sets of functional $\mathrm{T} 2 * \mathrm{w}$ images were acquired for each mouse with the Bruker 9.4T scanner. The $\mathrm{T} 2 \mathrm{w}$ images were acquired using a TurboRARE sequence: FOV $=16 \times 16 \mathrm{~mm}^{2}$, matrix $=256 \times$ 256 , in-plane resolution $=0.0625 \times 0.0625 \mathrm{~mm}^{2}$, slice number $=32$, slice thickness $=0.4 \mathrm{~mm}$, RAREfactor $=8$, TR/TE $=3200 \mathrm{~ms} / 33 \mathrm{~ms}$. Two sets of 
153 functional $\mathrm{T} 2 * \mathrm{w}$ data, acquired using a single-shot echo planar imaging (EPI)

154 sequence, consist of a high spatial resolution one (EPI01) and a high temporal

155 resolution one (EPI02). Parameters for EPI01 were: FOV $=15 \times 10.05 \mathrm{~mm}^{2}$,

156 matrix size $=100 \times 67$, in-plane resolution $=0.15 \times 0.15 \square \mathrm{mm}^{2}$, slice number $=$

15715 , slice thickness $=0.4 \mathrm{~mm}$, flip angle $=60^{\circ}, \mathrm{TR} / \mathrm{TE}=1500 \mathrm{~ms} / 15 \mathrm{~ms}$, repetitions

$158=256$. EPI02 images were acquired with the following parameters: FOV $=15 \times 12$

$159 \mathrm{~mm}^{2}$, matrix size $75 \times 60$, in-plane resolution $=0.2 \times 0.2 \mathrm{~mm}^{2}$, slice number $=10$,

160 slice thickness $=0.4 \mathrm{~mm}$, flip angle $=35^{\circ}, \mathrm{TR} / \mathrm{TE}=350 \mathrm{~ms} / 15 \mathrm{~ms}$, repetitions $=$ 1611100.

\subsection{U-Net}

The U-Net architecture is shown in Figure 1. It consists of one encoding and one decoding path with a skip connection. The skip connection, which connects the corresponding downsampling and upsampling stages, allows the model to integrate multi-scale information and better propagate the gradients for improved performance. Notably, the anatomical and functional images were usually acquired slice-by-slice with 2D sequence to span the whole brain in the fMRI study, resulting in an intra-slice spatial resolution that is significantly higher than inter-slice spatial

171 resolution. In such case, the conventional approach is to take the individual 2D slice 172 as input and predict the corresponding brain mask with 2D U-Net. However, the 2D 173 model ignores the inter-slice information. To capture more spatial feature

174 information while preserve intra-slice information, we took the multi-slice 




Figure 1 - Architecture of the 3D U-Net for skull stripping. Each blue box indicates a multi-channel feature map, and the number of channels is denoted on top of the box. Every white box indicates the copied feature map. Color-coded arrows denote the different operations.

2D images as an anisotropic 3D volume and used 3D U-Net model for training by only maxpooling and upsampling within the slice.

Every stage in both the encoding and decoding path is composed of two repeated $3 \quad 33$ convolutions, each of which is followed by a rectified linear unit (ReLU), and a 22 slice max-pooling layer (in the encoding path) or a $2 \times 2$ slice deconvolutional layer (in the decoding path). The number of downsampling and upsampling is set to three, and the channel number of first stage is set to 32 , being doubled at each downsampling step and then halved at each upsampling step. The output layer following the last decoding stage is a $1 \quad 1 \quad 1$ single channel convolutional layer with a sigmoid activation function, which transforms the feature representation to one segmentation map. 

from those of $\mathrm{T} 2 *_{\mathrm{w}}$ images, using the data from both modalities to train a network may degrade the segmentation performance. In this study, two models were trained separately with anatomical $\mathrm{T} 2 \mathrm{w}$ images and functional $\mathrm{T} 2 *_{\mathrm{w}}$ images based on the above 3D U-Net architecture. As functional T2*w data include multiple repetitions in each section, only the first repetition was used in our study. A total of 74 mice were randomly selected from the first dataset for training, and the remaining 10 mice were used for testing. All of the second dataset were also used as test data to verify the effectiveness of our model across different data sources. In the training phase, $80 \%$ of training data from 74 mice were selected randomly to train the model, and the remaining $20 \%$ was used to validate the model to avoid over-fitting. Furthermore, 198 data augmentation like flipping and rotating around three axes was also used to increase the diversity of the training dataset to improve the generalization of the models. 2016) and trained on a Nvidia Titan X GPU (12GB). The convolution parameters were randomly initialized from a normal distribution with mean value of zero and a standard deviation of 0.001 . Adam was used to optimize the training network

205 (Kingma and $\mathrm{Ba}, 2014$ ) with mini-batch size of two, and batch normalization (Ioffe 206 and Szegedy, 2015) was adopted to accelerate the network training. The maximal

207 number of training epochs was set to 100 . The learning rate started from $10^{-4}$ and 208 decayed by a factor of 0.99 every epoch. The loss function used to train the models 
was focal loss (Lin et al., 2020), which is designed to address class imbalance and can focus training on a sparse set of hard examples with large errors.

\subsection{Comparison of methods}

3D U-Net models were compared to two widely used methods for mouse brain extraction: RATS and SHERM. The parameters of both methods were carefully tuned to achieve the best performance for each dataset. For RATS (Oguz et al., 2014), the brain volume was set to $380 \mathrm{~mm}^{3}$ for both D1 and D2. In D1, the intensity threshold was set to 2.1 times of average intensity of each image for the T2w modality and 1.2 times for the $\mathrm{T} 2 * \mathrm{~W}$ modality. In $\mathrm{D} 2$, the threshold value was set to 1.3 times for the T2w and 0.6 times for the T2*w. For SHERM (Liu et al., 2020), the range of brain volume was set to $300-550 \mathrm{~mm}^{3}$. Except for the above parameters, all others were set to the default values for RATS and SHERM.

\subsection{Data pre- and post-processing}

All images were preprocessed before being fed into the models as follows. First, the N4bias field correction (Tustison et al., 2010) was applied to correct the intensity inhomogeneity for all images with the Python SimpleITK (Yaniv et al., 2018). Second, we resampled all data to a resolution of $0.0625 \times 0.0625 \mathrm{~mm}^{2}$ for the anatomical $\mathrm{T} 2 \mathrm{w}$ images and $0.25 \times 0.25 \mathrm{~mm}^{2}$ for the functional $\mathrm{T} 2 * \mathrm{w}$ images within the slice, leaving the inter-slice resolution unchanged. Then, all images were zero-padded to a size of $256 \times 256$ for the anatomical images and $64 \times 64$ for the 
231 functional images within slices. The padding was not performed in the inter-slice

232 direction when the slice number was larger than 20 , otherwise the slice number was 233 padded to 20 with edge values of the slice. Subsequently, the histogram of each

234 image was matched to a target histogram that was the average of all histograms of 235 the training dataset. Finally, each anisotropic volume data was normalized in the 236 range $[0,1]$.

237 After obtaining the binary mask from the network's probability output, the only 238 post-processing step was to identify the largest connected component and discard all 239 the others (disconnected ones) for the final brain mask.

\subsection{Evaluation methods}

Two types of assessment methods were used to evaluate the effectiveness of the

3D U-Net models. The first type measured the overlap of the predicted segmentation

Jaccard index, and the Hausdorff distance.

248 The Dice coefficient is defined as twice the size of the intersection of the two masks

249 divided by the sum of their sizes:

250 Dice $=\frac{2\left|M_{\text {manual }} \cap M_{\text {predicted }}\right|}{\left|M_{\text {manual }}\right|+\left|M_{\text {predicted }}\right|}(1)$

252 The Jaccard index is defined as the size of the intersection of the two masks divided 
by the size of their union:

$254 \quad$ Jaccard $=\frac{\left|M_{\text {manual }} \cap M_{\text {predicted }}\right|}{\left|M_{\text {manual }} \cup M_{\text {predicted }}\right|}(2)$

The Hausdorff distance (Huttenlocher et al., 1993) between two finite point sets is

257

258

259

260

261

262

263

264

265

266

267

268

269

270

271

272

273

274

defined as:

Hausdorff $=\max \left\{\sup _{x \in X} \inf _{y \in Y}\|x-y\|, \sup _{y \in Y} \inf _{x \in X}\|x-y\|\right\}$,

where $X$ and $Y$ denote the boundaries of the predicted segmentation mask and the manual segmentation mask, respectively. To exclude possible outliers, the Hausdorff distance is redefined as the 95th percentile distance instead of the maximum in our study.

The second assessment involves evaluating the impact of automatic skull stripping with 3D U-Net models on the final result of the fMRI analysis. The fMRI data were preprocessed with the common pipeline after skull stripping and then analyzed with seed-based analysis (Chan et al., 2017; Wang et al., 2019) and group independent component analysis (group ICA) (Mechling et al., 2014; Zerbi et al., 2015), which are two widely used methodologies in fMRI studies. Seed-based analysis requires creating a seed region first and then generates a seed-to-brain connectivity map by calculating the Pearson's correlation coefficient (CC) between the BOLD time course of the seed and those of all other voxels in the brain. Group ICA is a data-driven method for the blind source separation of fMRI data, which is implemented in the group ICA of fMRI toolbox (GIFT) (Rachakonda et al., 2007). The Pearson's correlation coefficient was calculated between the time courses of each pair of 
components extracted from the group ICA.

276

\section{$277 \quad 3$ Results}

278

Figure 2 illustrates the segmentation results of RATS, SHERM, and the 3D U-Net

279

model on the T2w images from one mouse in D1 (figure 2a) and from two mice of auditory (b) and somatosensory (c) stimulation in D2. As shown in Figure 2a, all three methods successfully extracted most brain tissue. By comparison, the segmentation contours of the 3D U-Net model and SHERM were smoother and closer to the ground truth than those of RATS. However, the brain mask predicted by SHERM misaligned with the ground truth at the sharp-angled corner, while the 3D U-Net model still performed well in these locations. As shown in Figure $2 \mathrm{~b}$ and $2 \mathrm{c}$, the segmentation performance on T2w images of D2 was degraded for RATS and SHERM, compared to that of D1. The performance degradation might be attributed to T2w images of D2 having lower SNR and image contrast than those of D1. Specifically, the RATS produced a brain mask with a rough boundary, while the SHERM evidently overestimated the entire brain volume on D2. However, the 3D U-Net model still performed well on both D2 and D1.

Figure 3 shows the segmentation results for three representative slices of RATS, SHERM, and the 3D U-Net model on T2*w images from one mouse in D1 (Figure 3a)

294 and from four mice in D2 with high spatial resolution (EPI01) (Figure 3b_1, 3c_1)

295 and high temporal resolution (EPI02) (Figure 3b_2, 3c_2). The SHERM 296 overestimated the brain volume, especially for the D2. The RATS and 3D U-Net 
297 model performed better than SHERM in both D1 and D2. However, the brain

298 boundaries predicted by the RATS were misaligned with the ground-truth boundaries

299 in the areas with severe distortions or signal losses (white arrows), while the 3D

300 U-Net model still had an accurate alignment on these locations.
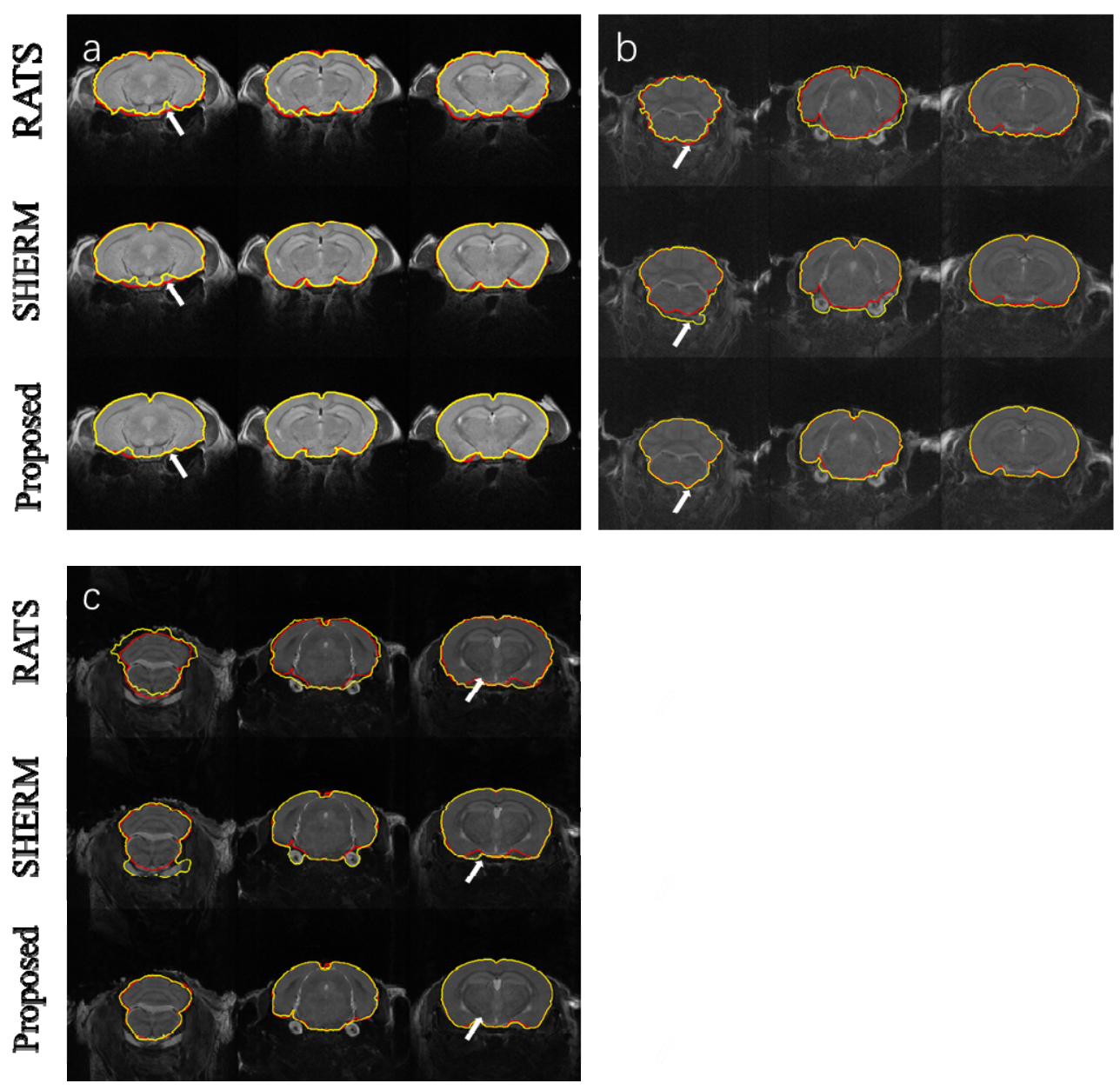

Figure 2 - Example segmentation comparison for T2w images from one mouse in D1 (a) and from two mice with auditory (b) and somatosensory (c) stimulation in D2. Red lines show the contours of ground truth; yellow lines show automatically computed brain masks by RATS, SHERM, and the 3D U-Net model. White arrows point to the rough boundary, where the 3D U-Net model performed better than RATS and SHERM. 

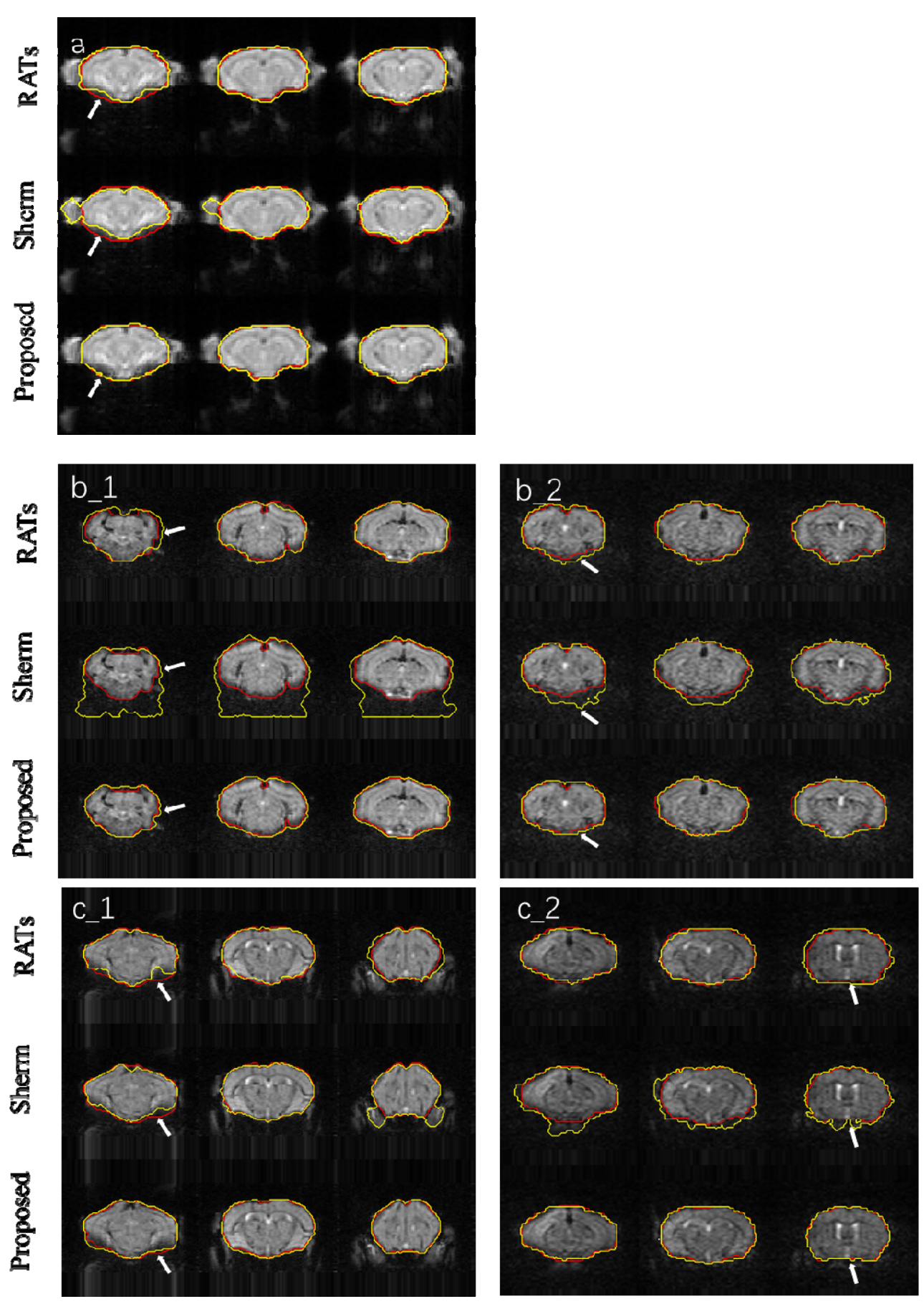

Figure 3 - Example segmentations comparison for $\mathrm{T} 2 * \mathrm{w}$ images from one mouse in D1 (a) and from two mice with auditory stimulation (b) and two mice with somatosensory stimulation $(\mathbf{c})$ in D2; b_1, c_1 represent EPI01, and b_2, c_2 represent EPI02. Red lines show the contours of ground truth; yellow lines show automatically computed brain masks by RATS, SHERM, and the 3D U-Net model. White arrows point to the rough boundary, where 3D U-Net models performed better than RATS and SHERM. 
Tables 1 and Table 2 show the quantitative assessment of RATS, SHERM, and 3D U-Net models for $\mathrm{T} 2 \mathrm{w}$ images and $\mathrm{T} 2{ }^{*} \mathrm{w}$ images, respectively. The 3D U-Net models yielded highest mean values of the Dice and Jaccard index, and lowest mean

307 values of the Hausdorff distance in both $\mathrm{T} 2 \mathrm{w}$ and $\mathrm{T} 2 * \mathrm{w}$ images. In addition, the standard deviations of all three metrics were lowest for the 3D U-Net models in most cases, except for the Hausdorff distance on T2w images of D2_PART2, and the Dice and Jaccard index on T2*w images of both D1 and D2_PART2_EPI01, which were

311 slightly higher than those of RATS. The above quantitative results indicate that the 3D

312 U-Net models exhibit a high accuracy and stability.

313 Figure 4 compares the results of seed-based analysis from one mouse in the test

314 data of D1 with automatic skull stripping by the 3D U-Net models and manual brain

315 extraction. The seeds (2 $\square \times \square 2$ voxels) were positioned in the dorsal striatum (dStr),

316 somatosensory barrel field cortex (S1BF), secondary somatosensory cortex (S2), and

317 ventral striatum (vStr). The CC maps with our model were similar to those with

318 manual brain extraction. We also plotted scatter plots, where the horizontal axis

319 represents the values of $\mathrm{CC}$ maps with predicted masks, and the vertical axis

320 represents the values of CC maps with the manual mask. Each point represents a pair

321 of the two values at the same pixel location. All points were concentrated on the

322 diagonal with $\mathrm{R}^{2}=1$. The above results indicate that the CC maps with automatic

323 skull stripping by 3D U-Net models were identical to those with manual brain 324 extraction.

325 Figure 5 compared the four components of group ICA analysis with automatic 
Table 1 - Mean and standard deviation of Dice, Jaccard index, and Hausdorff distance 328 evaluating the RATS, SHERM, and 3D U-Net model for T2w images in different 329 datasets. Bold values indicate the best results.

\begin{tabular}{|c|c|c|c|c|}
\hline Dataset & Method & Dice & $\begin{array}{l}\text { Jaccard } \\
\text { index }\end{array}$ & $\begin{array}{l}\text { Hausdoff } \\
\text { distance }\end{array}$ \\
\hline \multirow[t]{3}{*}{ D1 } & RATs & $0.9377 \pm 0.0036$ & $0.8826 \pm 0.0064$ & $8.97 \pm 0.99$ \\
\hline & Sherm & $0.9767 \pm 0.0028$ & $0.9545 \pm 0.0054$ & $6.02 \pm 1.32$ \\
\hline & Proposed & $0.9898 \pm 0.0013$ & $0.9800 \pm 0.0025$ & $3.53 \pm 0.86$ \\
\hline \multirow{3}{*}{$\begin{array}{l}\text { D2_PART1 } \\
\text { (auditory) }\end{array}$} & RATs & $0.9404 \pm 0.0038$ & $0.8875 \pm 0.0068$ & $8.77 \pm 1.61$ \\
\hline & Sherm & $0.9686 \pm 0.0079$ & $0.9392 \pm 0.0147$ & $20.28 \pm 8.55$ \\
\hline & Proposed & $0.9842 \pm 0.0014$ & $0.9690 \pm 0.0027$ & $6.04 \pm 1.08$ \\
\hline \multirow{3}{*}{$\begin{array}{c}\text { D2_PART2 } \\
\text { (somatosensory) }\end{array}$} & RATs & $0.9442 \pm 0.0049$ & $0.8943 \pm 0.0087$ & $9.69 \pm \mathbf{1 . 6 1}$ \\
\hline & Sherm & $0.9735 \pm 0.0048$ & $0.9484 \pm 0.0091$ & $16.17 \pm 6.04$ \\
\hline & Proposed & $0.9845 \pm 0.0023$ & $0.9696 \pm 0.0044$ & $\mathbf{6 . 9 5} \pm 1.96$ \\
\hline
\end{tabular}

330

331

332

333

334

335

336

337

338

339

340

341

342

343

skull stripping by the 3D U-Net models and manual brain extraction. The presented four components in Figure 5 correspond to the same regions used in the seed-based analysis (S1BF, S2, dStr, vStr). The component maps with 3D U-Net models for skull stripping were visually similar to those with manual brain extraction. The points in the scatter plots were concentrated on the diagonal with $\mathrm{R}^{2}=1$. These results indicate that the component maps of group ICA with automatic skull stripping by 3D U-Net models are highly consistent with those with manual brain extraction. Group ICA analysis with 3D U-Net models generated the same 32 independent components as those with manual brain extraction. The regions corresponding to each component are shown in Supplementary Table S1. 
344 Table 2 - Mean and standard deviation of Dice, Jaccard index, and Hausdorff distance 345 evaluating the RATS, SHERM, and 3D U-Net model for T2*w images in different 346 datasets. Bold values indicate the best results.

\begin{tabular}{|c|c|c|c|c|}
\hline Dataset & Method & Dice & Jaccard index & $\begin{array}{l}\text { Hausdoff } \\
\text { distance }\end{array}$ \\
\hline \multirow[t]{3}{*}{ D1 } & RATs & $0.9467 \pm \mathbf{0 . 0 0 2 8}$ & $0.8989 \pm \mathbf{0 . 0 0 5 1}$ & $2.98 \pm 0.48$ \\
\hline & SHERM & $0.9070 \pm 0.0125$ & $0.8301 \pm 0.0211$ & $5.17 \pm 1.82$ \\
\hline & Proposed & $\mathbf{0 . 9 6 4 4} \pm 0.0044$ & $\mathbf{0 . 9 3 1 3} \pm 0.0083$ & $2.95 \pm 0.56$ \\
\hline \multirow{3}{*}{$\begin{array}{c}\text { D2_PART1_EPI01 } \\
\text { (auditory) }\end{array}$} & RATs & $0.9504 \pm \mathbf{0 . 0 0 3 1}$ & $0.9057 \pm \mathbf{0 . 0 0 5 6}$ & $4.49 \pm 0.78$ \\
\hline & SHERM & $0.8306 \pm 0.1108$ & $0.7260 \pm 0.1665$ & $17.18 \pm 9.04$ \\
\hline & Proposed & $\mathbf{0 . 9 6 4 4} \pm 0.0044$ & $\mathbf{0 . 9 3 1 3} \pm 0.0083$ & $2.95 \pm 0.56$ \\
\hline \multirow{3}{*}{$\begin{array}{c}\text { D2_PART1_EPI02 } \\
\text { (auditory) }\end{array}$} & RATs & $0.9453 \pm 0.0206$ & $0.8969 \pm 0.0353$ & $3.56 \pm 1.54$ \\
\hline & SHERM & $0.8887 \pm 0.0388$ & $0.8018 \pm 0.0609$ & $9.90 \pm 3.53$ \\
\hline & Proposed & $0.9663 \pm 0.0078$ & $0.9350 \pm 0.0144$ & $2.57 \pm 0.94$ \\
\hline \multirow{3}{*}{$\begin{array}{l}\text { D2_PART2_EPI01 } \\
\text { (somatosensory) }\end{array}$} & RATs & $0.9484 \pm 0.0064$ & $0.9019 \pm 0.0116$ & $5.52 \pm 1.06$ \\
\hline & SHERM & $0.8939 \pm 0.1040$ & $0.8226 \pm 0.1538$ & $12.21 \pm 7.63$ \\
\hline & Proposed & $0.9694 \pm 0.0037$ & $0.9406 \pm 0.0070$ & $3.30 \pm 0.59$ \\
\hline \multirow[t]{3}{*}{ D2_PART2_EPI02 } & RATs & $0.9516 \pm 0.0076$ & $0.9077 \pm 0.0139$ & $3.74 \pm 0.75$ \\
\hline & SHERM & $0.9284 \pm 0.0241$ & $0.8674 \pm 0.0411$ & $6.70 \pm 1.87$ \\
\hline & Proposed & $0.9650 \pm 0.0068$ & $0.9326 \pm 0.0126$ & $2.83 \pm 0.75$ \\
\hline
\end{tabular}

347

348

349

350

Figure 6 shows the functional network connectivity (FNC) correlations for one mouse and the average FNC correlation across 10 mice between each pair of 32 regions extracted from group ICA analysis. Each correlation value in FNC matrixes 

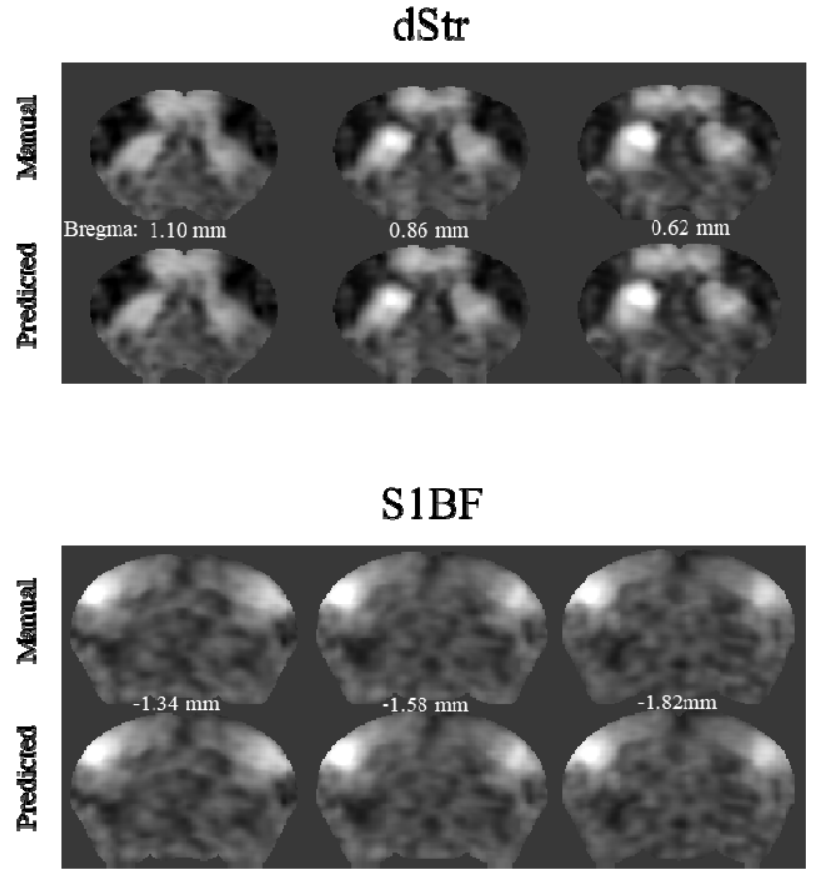

S2

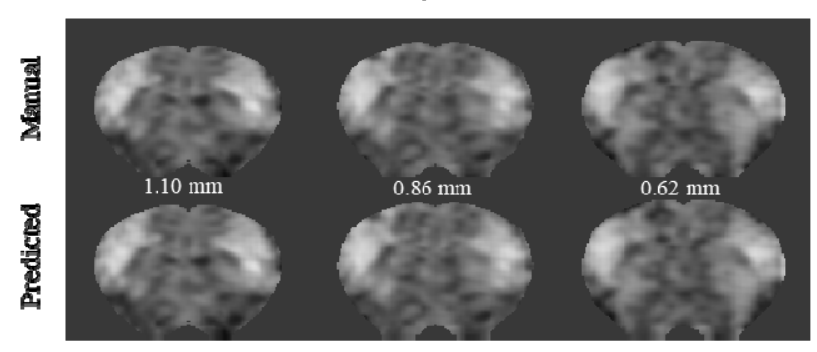

vStr

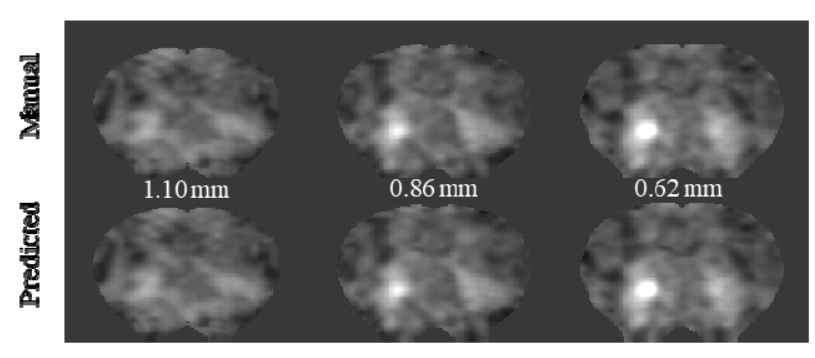

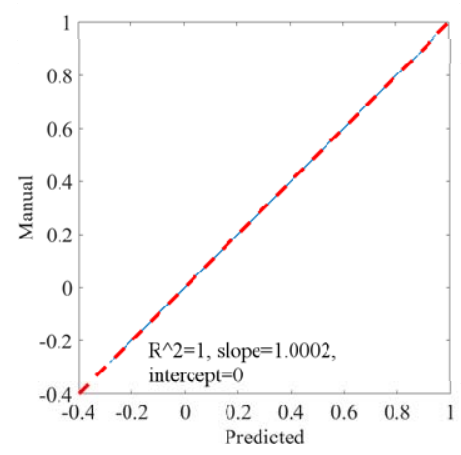


Figure 4 - Exemplary results of seed-based analysis for one mouse in the test data of D1. The selected seed regions were S1BF, S2, dStr, and vStr. The left column illustrates the $\mathrm{CC}$ maps with manual brain extraction and automatic skull stripping by the 3D U-Net models for each seed region. The corresponding right column illustrates the scatters, where each point represents a pair of the two values from different CC maps with manual brain extraction and automatic skull stripping by the 3D U-Net models, at the same pixel location. 

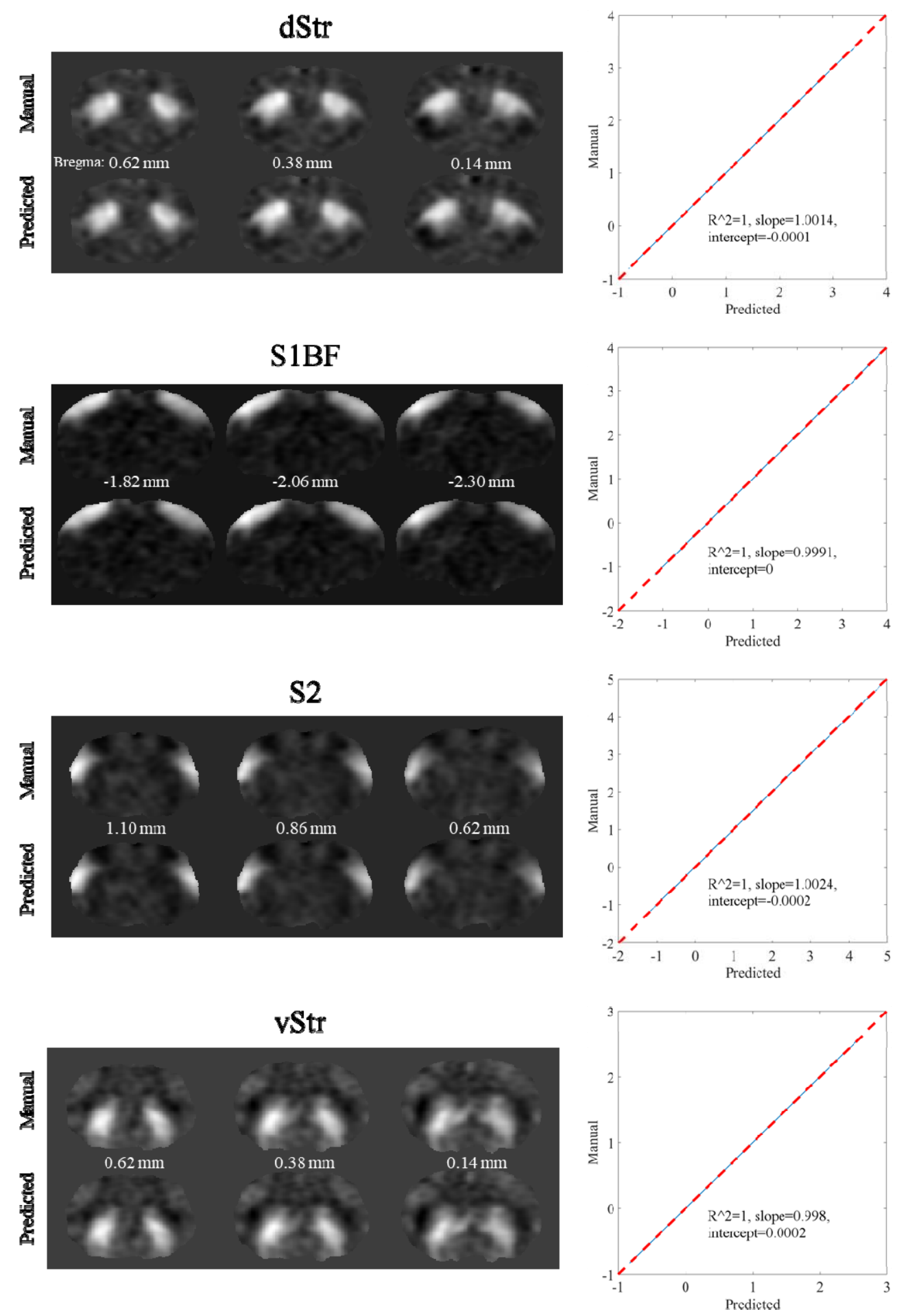

Figure 5 - Example results of ICA analysis for test data of D1. Four selected components extracted by group ICA matching the four seed regions in seed-based analysis are shown in each row. The left column illustrates the component maps with manual brain extraction and automatic skull stripping by 3D U-Net models. The right column illustrates the scatters, where each point represents a pair of the two values from different component maps with manual brain extraction and automatic skull stripping by the 3D U-Net models, respectively, at the same pixel location. 
353

354 with automatic skull stripping by 3D U-Net models was identical to that obtained with manual brain extraction. The points in the scatter plots are concentrated on the diagonal with $\mathrm{R}^{2}=1$, which indicates that the use of the 3D U-Net models for skull stripping in the fMRI analysis pipeline does not affect the final results of group ICA based FC analysis. The FNC correlations of the other nine mice are shown in Supplementary Figure S1.

\section{Discussion}

To the best of our knowledge, this is the first study investigating the feasibility of 3D U-Net for mouse skull stripping from brain functional $(\mathrm{T} 2 * \mathrm{w})$ images and the impact of automatic skull stripping on the final fMRI analysis. Results indicate that the 3D U-Net model performed well on both anatomical (T2w) and functional $\left(\mathrm{T} 2{ }^{*} \mathrm{w}\right)$ images and achieved state-of-the-art performance in mouse brain extraction. The fMRI results with automatic brain extraction using 3D U-Net models are nearly identical with those with manual brain extraction. Thus, the manual brain extraction in the fMRI pre-processing pipeline can be replaced by the proposed automatic skull-stripping method.

The 3D U-Net models were tested on not only interior but also exterior datasets. Notably, the exterior datasets were acquired with different acquisition parameters on a scanner with different field strength from another MRI center. The results show that 3D U-Net models had high segmentation accuracy that is comparable between interior and exterior datasets. This demonstrates that the developed method has high reliability 
FNC
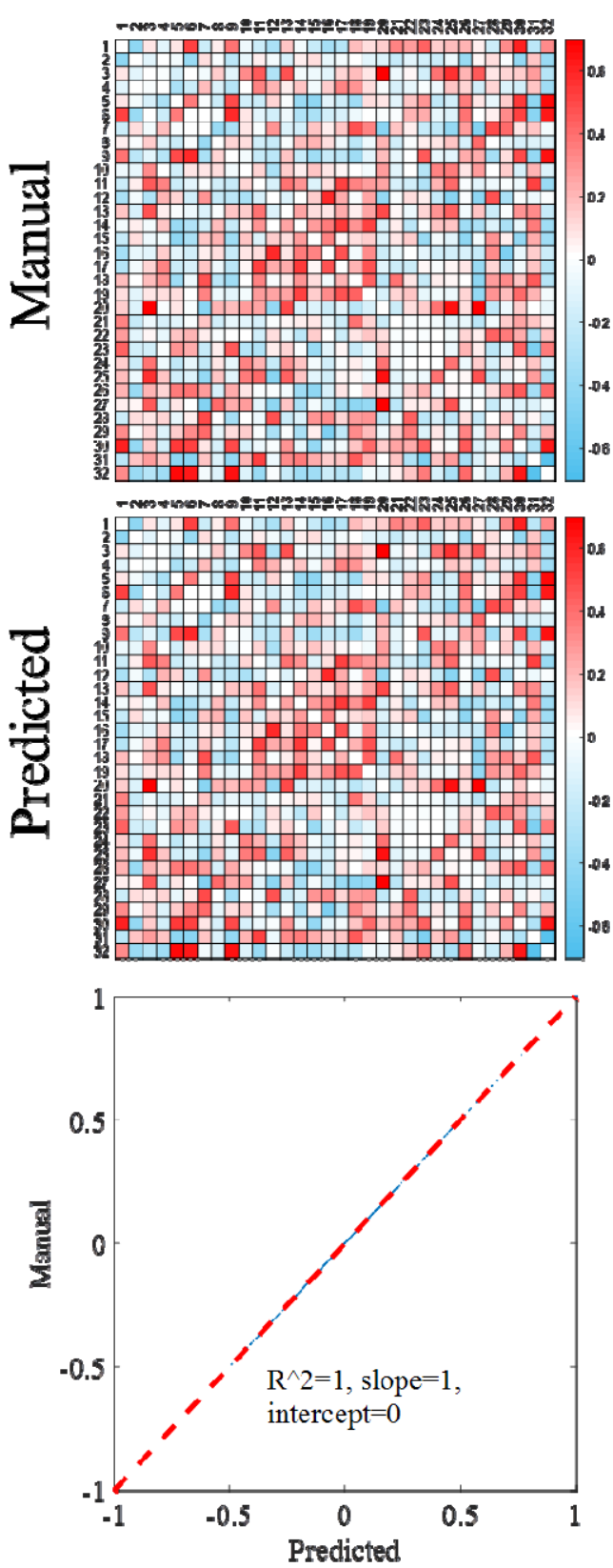

Average FNC
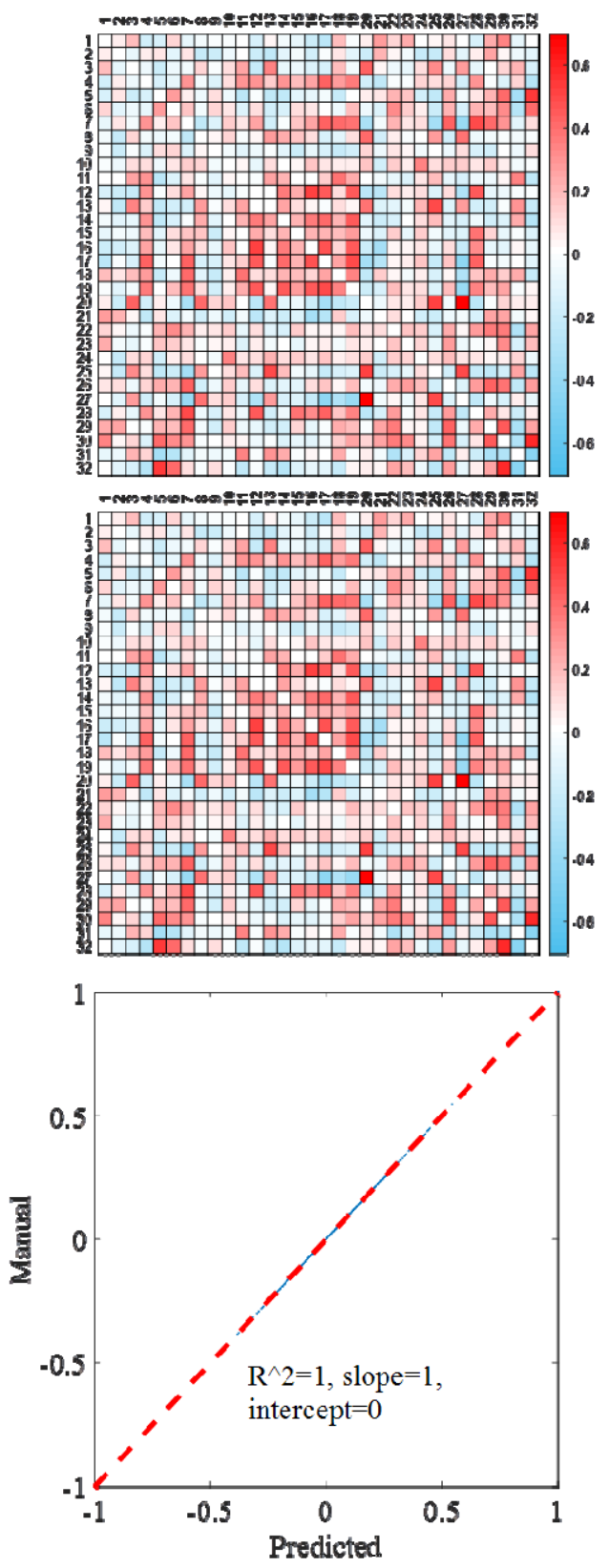

Figure 6 -Functional connectivity between the independent components extracted from group ICA analysis for the test data of D1. The left column illustrates the FNC matrixes from one mouse, and the right column illustrates the average FNC matrixes across 10 mice. Scatter plots are shown in the bottom row, where each point represents the difference between two values from two FNC matrixes with manual brain extraction and automatic skull stripping by the 3D U-Net models, at the same pixel location. 
376 and excellent generalization ability. The 3D U-Net models also outperformed two

377 widely used brain extractions for rodent (SHERM and RATS). SHERM has the

378 second best performance in $\mathrm{T} 2 \mathrm{w}$ images (Figure 2), while it has the worst

379 performance in $\mathrm{T} 2 * \mathrm{w}$ images (Figure 3 ). The reason for this is that the poor quality of

$380 \mathrm{~T} 2 *_{\mathrm{w}}$ images makes it difficult to match the shape of the brain template. Although

381 RATS has stable performance across different datasets and modalities, its

382 segmentation accuracy (Dice $<0.945$ in $\mathrm{T} 2 \mathrm{w}$ images and Dice $<0.952$ in $\mathrm{T} 2 * \mathrm{w}$

383 images) is consistently lower than in our method (Dice $>0.984$ in T2w images and

384 Dice $>0.964$ in $\mathrm{T} 2 * \mathrm{w}$ images).

385 There are several related reports on using U-Net for mouse skull stripping (De

386 Feo et al., 2021; Hsu et al., 2020; Thai et al., 2019). Compared with the model

387 adopted by Hsu et al. (2020), the segmentation accuracy of our models is relatively

388 higher in both $\mathrm{T} 2 \mathrm{w}$ and $\mathrm{T} 2 * \mathrm{w}$ images. The first reason is that we used 3D U-Net for

389 mouse skull stripping, while Hsu et al. used 2D U-Net. The second reason is that we

390 trained the U-Net models separately for T2- and T2*w images. The performance of

391 our 3D U-Net model is comparable to that of the 3D model adopted by De Feo et al.

392 (2021) on T2w anatomical images. We also applied the 3D U-Net for brain extraction

393 from $\mathrm{T} 2 * \mathrm{w}$ functional images. The multi-task U-Net developed by De Feo et al. can

394 hardly be applied to functional images, because it is difficult to delineate different

395 brain regions in functional images due to their low spatial resolution, contrast,

396 signal-to-noise ratio, and severe distortion.

397 It is essential to guarantee that automatic skull-stripping method does not alter 
398

399

400

401

402

403

404

405

406

407

408

409

410

411

412

413

414

415

416

417

418

419

fMRI analysis results. Thus, we not only evaluated the segmentation accuracy, but also investigated the effect of automatic segmentation on fMRI analysis results. The fMRI analysis results with automatic skull stripping by 3D U-Net models are identical to those with manual skull stripping. This finding demonstrates that the 3D U-Net based method can replace manual skull stripping and facilitate the establishment of the automated fMRI analysis pipeline for the mouse model.

With respect to the computational cost, the 3D U-Net based method proves to be time efficient. The computation time of the 3D U-Net method was approximately $3 \mathrm{~s}$ for a T2w volume data with a size of $256 \times 256 \times 20$, and $0.5 \mathrm{~s}$ for $\mathrm{T} 2 * \mathrm{w}$ volume data with a size of $64 \times 64 \times 20$. In comparison, the computation time of SHERM is $780 \mathrm{~s}$ and $3 \mathrm{~s}$ for $\mathrm{T} 2 \mathrm{w}$ and $\mathrm{T} 2 * \mathrm{w}$ images, respectively; the computation time of RATS is $10 \mathrm{~s}$ for $\mathrm{T} 2 \mathrm{w}$ images and $3 \mathrm{~s}$ for $\mathrm{T} 2 * \mathrm{w}$ images. All test procedures were run on a server with a Linux 4.15.0 system, an Intel(R) Xeon(R) E5-2667 8-core CPU, and 256 GB RAM.

There are two limitations in our current work. First, the segmentation accuracy of the developed 3D U-Net model on functional images is still relatively lower than that on anatomical images, because of the poor image quality of the functional images. Utilizing the cross-modality information between anatomical and functional images may further improve the accuracy of skull stripping on functional images. Second, the developed 3D U-Net model was only trained and validated on adult C57BL/6 mice, and cannot be directly applied to brain MR images from different mouse types and ages. To address this problem, the model need to be retrained by including more 
420

421

422

423

424

425

426

427

428

429

430

431

432

433

434

435

436

\section{Acknowledgments}

438

439

440

Zhu et al., 2021).

\section{Conclusion} fMRI data processing.

manually labeled data from mice with varying types and ages. Labeling data is time-consuming and labor-intensive, and another potential approach to reduce the amount of labeled data is to utilize transfer learning (Long et al., 2015; Yu et al., 2019;

We investigated an automatic skull-stripping method based on 3D U-Net for mouse fMRI analysis. The 3D U-Net based method achieves state-of-the-art performance on both $\mathrm{T} 2 \mathrm{w}$ and $\mathrm{T} 2{ }^{*} \mathrm{w}$ images in terms of the segmentation accuracy. Identical results between mouse fMRI analysis using manual and automatic skull stripping demonstrates that the 3D U-Net model has a great potential to replace manual labeling in the mouse fMRI analysis pipeline. Hence, skull stripping by the 3D U-Net model will facilitate the establishment of an automatic pipeline of mouse

This work receives support from the National Natural Science Foundation of China (61971214, 81871349); the Natural Science Foundation of Guangdong Province (2019A1515011513); the Guangdong-Hong Kong-Macao Greater Bay Area Center 
441 for Brain Science and Brain-Inspired Intelligence Fund (2019022); the

442 Technology R\&D Program of Guangdong (2017B090912006); the National Key

443 R\&D Program of China (2019YFC0118702).

444

\section{References}

446 Abadi, M., Barham, P., Chen, J., Chen, Z., Davis, A., Dean, J., Devin, M., Ghemawat, S., Irving,

447 G., Isard, M., 2016. Tensorflow: A system for large-scale machine learning. 12th \{USENIX\}

448 symposium on operating systems design and implementation (\{OSDI\} 16), pp. 265-283.

449 Chan, R.W., Leong, A.T.L., Ho, L.C., Gao, P.P., Wong, E.C., Dong, C.M., Wang, X., He, J.,

450 Chan, Y.S., Lim, L.W., Wu, E.X., 2017. Low-frequency hippocampal-cortical activity drives

451 brain-wide resting-state functional MRI connectivity. Proc Natl Acad Sci U S A 114,

452 E6972-E6981. https://doi.org/10.1073/pnas.1703309114

453 Chen, X., Tong, C., Han, Z., Zhang, K., Bo, B., Feng, Y., Liang, Z., 2020. Sensory evoked fMRI

454 paradigms in awake mice. Neuroimage 204, 116242.

455 https://doi.org/10.1016/j.neuroimage.2019.116242

456 Chou, N., Wu, J., Bai Bingren, J., Qiu, A., Chuang, K.H., 2011. Robust automatic rodent brain

457 extraction using 3-D pulse-coupled neural networks (PCNN). IEEE Trans Image Process 20,

458 2554-2564. https://doi.org/10.1109/TIP.2011.2126587

459 D'Esposito, M., Aguirre, G.K., Zarahn, E., Ballard, D., Shin, R.K., Lease, J., 1998. Functional

460 MRI studies of spatial and nonspatial working memory. Brain Res Cogn Brain Res 7, 1-13.

461 https://doi.org/10.1016/s0926-6410(98)00004-4

462 De Fauw, J., Ledsam, J.R., Romera-Paredes, B., Nikolov, S., Tomasev, N., Blackwell, S., 
463

464

465

466

467

468

469

470

471

472

473

474

475

476

477

478

479

480

481

482

483

484

Askham, H., Glorot, X., O'Donoghue, B., Visentin, D., 2018. Clinically applicable deep learning

for diagnosis and referral in retinal disease. Nature medicine $24,1342-1350$.

https://doi.org/10.1038/s41591-018-0107-6

De Feo, R., Shatillo, A., Sierra, A., Valverde, J.M., Grohn, O., Giove, F., Tohka, J., 2021.

Automated joint skull-stripping and segmentation with Multi-Task U-Net in large mouse brain

MRI databases. Neuroimage 229, 117734. https://doi.org/10.1016/j. neuroimage.2021.117734

Eskildsen, S.F., Coupe, P., Fonov, V., Manjon, J.V., Leung, K.K., Guizard, N., Wassef, S.N.,

Ostergaard, L.R., Collins, D.L., Alzheimer's Disease Neuroimaging, I., 2012. BEaST: brain

extraction based on nonlocal segmentation technique. Neuroimage 59, 2362-2373.

https://doi.org/10.1016/j.neuroimage.2011.09.012

Guo, Z., Li, X., Huang, H., Guo, N., Li, Q., 2019. Deep learning-based image segmentation on multimodal medical imaging. IEEE Transactions on Radiation and Plasma Medical Sciences 3, 162-169. https://doi.org/10.1109/TRPMS.2018.2890359

Hsu, L.M., Wang, S., Ranadive, P., Ban, W., Chao, T.H., Song, S., Cerri, D.H., Walton, L.R., Broadwater, M.A., Lee, S.H., Shen, D., Shih, Y.I., 2020. Automatic Skull Stripping of Rat and Mouse Brain MRI Data Using U-Net. Front Neurosci 14, 568614.

https://doi.org/10.3389/fnins.2020.568614

Huang, M., Yang, W., Jiang, J., Wu, Y., Zhang, Y., Chen, W., Feng, Q., Alzheimer's Disease

Neuroimaging, I., 2014. Brain extraction based on locally linear representation-based

classification. Neuroimage 92, 322-339. https://doi.org/10.1016/j.neuroimage.2014.01.059

Huttenlocher, D.P., Klanderman, G.A., Rucklidge, W.J., 1993. Comparing images using the

Hausdorff distance. IEEE Transactions on pattern analysis and machine intelligence 15, 
485

486

487

488

489

490

491

492

493

494

495

496

497

498

499

500

501

502

503

504

505

506
850-863. https://doi.org/10.1109/34.232073

loffe, S., Szegedy, C., 2015. Batch normalization: Accelerating deep network training by

reducing internal covariate shift. pp. 448-456. arXiv:1502:03167

Jonckers, E., Van Audekerke, J., De Visscher, G., Van der Linden, A., Verhoye, M., 2011.

Functional connectivity fMRI of the rodent brain: comparison of functional connectivity

networks in rat and mouse. PLoS One 6, e18876.

https://doi.org/10.1371/journal.pone.0018876

Kingma, D.P., Ba, J., 2014. Adam: A method for stochastic optimization. arXiv:1412.6980.

Kleesiek, J., Urban, G., Hubert, A., Schwarz, D., Maier-Hein, K., Bendszus, M., Biller, A., 2016.

Deep MRI brain extraction: A 3D convolutional neural network for skull stripping. Neuroimage

129, 460-469. https://doi.org/10.1016/j.neuroimage.2016.01.024

Lake, E.M.R., Ge, X., Shen, X., Herman, P., Hyder, F., Cardin, J.A., Higley, M.J., Scheinost, D.,

Papademetris, X., Crair, M.C., Constable, R.T., 2020. Simultaneous cortex-wide fluorescence

$\mathrm{Ca}(2+)$ imaging and whole-brain fMRI. Nat Methods 17, 1262-1271.

https://doi.org/10.1038/s41592-020-00984-6

Lee, J.H., Durand, R., Gradinaru, V., Zhang, F., Goshen, I., Kim, D.S., Fenno, L.E.,

Ramakrishnan, C., Deisseroth, K., 2010. Global and local fMRI signals driven by neurons

defined optogenetically by type and wiring. Nature 465, 788-792.

https://doi.org/10.1038/nature09108

Lee, M.H., Smyser, C.D., Shimony, J.S., 2013. Resting-state fMRI: a review of methods and

clinical applications. AJNR Am J Neuroradiol 34, 1866-1872.

https://doi.org/10.3174/ajnr.A3263 
507 Li, L., Zhao, X., Lu, W., Tan, S., 2020. Deep learning for variational multimodality tumor

508 segmentation in PET/CT. Neurocomputing 392, 277-295.

509 https://doi.org/10.1016/j.neucom.2018.10.099

510 Lin, T.Y., Goyal, P., Girshick, R., He, K., Dollar, P., 2020. Focal Loss for Dense Object

511 Detection. IEEE Trans Pattern Anal Mach Intell 42, 318-327.

512 https://doi.org/10.1109/TPAMI.2018.2858826

513 Liu, Y., Unsal, H.S., Tao, Y., Zhang, N., 2020. Automatic Brain Extraction for Rodent MRI

514 Images. Neuroinformatics 18, 395-406. https://doi.org/10.1007/s12021-020-09453-z

515 Long, M., Cao, Y., Wang, J., Jordan, M., 2015. Learning Transferable Features with Deep

516 Adaptation Networks. In: Francis, B., David, B. (Eds.), Proceedings of the 32nd International

517 Conference on Machine Learning. PMLR, Proceedings of Machine Learning Research, pp.

518 97--105.

519 Mechling, A.E., Hubner, N.S., Lee, H.L., Hennig, J., von Elverfeldt, D., Harsan, L.A., 2014.

520 Fine-grained mapping of mouse brain functional connectivity with resting-state fMRI.

521 Neuroimage 96, 203-215. https://doi.org/10.1016/j.neuroimage.2014.03.078

522 Oguz, I., Zhang, H., Rumple, A., Sonka, M., 2014. RATS: Rapid Automatic Tissue

523 Segmentation in rodent brain MRI. J Neurosci Methods 221, 175-182.

$524 \quad$ https://doi.org/10.1016/j.jneumeth.2013.09.021

525 Perez-Cervera, L., Carames, J.M., Fernandez-Molla, L.M., Moreno, A., Fernandez, B.,

526 Perez-Montoyo, E., Moratal, D., Canals, S., Pacheco-Torres, J., 2018. Mapping Functional

527 Connectivity in the Rodent Brain Using Electric-Stimulation fMRI. Methods Mol Biol 1718,

528 117-134. https://doi.org/10.1007/978-1-4939-7531-0_8 
529

530

531

532

533

534

535

536

537

538

539

540

541

542

543

544

545

546

547

548

549

550

Rachakonda, S., Egolf, E., Correa, N., Calhoun, V., 2007. Group ICA of fMRI toolbox (GIFT)

manual. Dostupné z http://www.nitrc.org/docman/view.php/55/295/v1.3d_GIFTManual

Ronneberger, O., Fischer, P., Brox, T., 2015. U-net: Convolutional networks for biomedical

image segmentation. International Conference on Medical image computing and

computer-assisted intervention. Springer, pp. 234-241.

https://doi.org/10.1007/978-3-319-24574-4_28

Roy, S., Knutsen, A., Korotcov, A., Bosomtwi, A., Dardzinski, B., Butman, J.A., Pham, D.L., 2018. A deep learning framework for brain extraction in humans and animals with traumatic

brain injury. 2018 IEEE 15th International Symposium on Biomedical Imaging (ISBI 2018).

IEEE, pp. 687-691. https://doi.org/10.1109/ISBI.2018.8363667

Segonne, F., Dale, A.M., Busa, E., Glessner, M., Salat, D., Hahn, H.K., Fischl, B., 2004. A

hybrid approach to the skull stripping problem in MRI. Neuroimage 22, 1060-1075.

https://doi.org/10.1016/j.neuroimage.2004.03.032

Smith, S.M., 2002. Fast robust automated brain extraction. Hum Brain Mapp 17, 143-155.

https://doi.org/10.1002/hbm.10062

Suk, H.-I., Lee, S.-W., Shen, D., Initiative, A.s.D.N., 2017. Deep ensemble learning of sparse regression models for brain disease diagnosis. Medical image analysis 37, 101-113.

https://doi.org/10.1016/j.media.2017.01.008

Sun, L., Zhang, S., Chen, H., Luo, L., 2019. Brain tumor segmentation and survival prediction using multimodal MRI scans with deep learning. Frontiers in neuroscience 13, 810.

https://doi.org/10.3389/fnins.2019.00810

Szegedy, C., Liu, W., Jia, Y., Sermanet, P., Anguelov, S.R.D., Erhan, D., Vanhoucke, V., 
551 Rabinovich, A, 2015. Going deeper with convolutions. 2015 IEEE Conference on Computer

552 Vision and Pattern Recognition (CVPR), 1-9. https://doi.org/10.1109/CVPR.2015.7298594

553 T. Wood, D.L., S. Williams, 2013. rBET: Making BET work for Rodent Brains. Proc Intl Soc

554 Mag Reson Med, p. 2706.

555 Thai, A, Bui, V., Reyes, L., Chang, L., 2019. Using Deep Convolutional Neural Network for

556 Mouse Brain Segmentation in DT-MRI. 2019 IEEE International Conference on Big Data (Big

557 Data). https://doi.org/10.1109/BigData47090.2019.9005976

558 Tustison, N.J., Avants, B.B., Cook, P.A., Zheng, Y., Egan, A., Yushkevich, P.A., Gee, J.C.,

559 2010. N4ITK: improved N3 bias correction. IEEE Trans Med Imaging 29, 1310-1320.

$560 \quad$ https://doi.org/10.1109/TMI.2010.2046908

561 Wang, X., Leong, A.T.L., Chan, R.W., Liu, Y., Wu, E.X., 2019. Thalamic low frequency activity

562 facilitates resting-state cortical interhemispheric MRI functional connectivity. Neuroimage 201,

563 115985. https://doi.org/10.1016/j.neuroimage.2019.06.063

564 Wehrl, H.F., Martirosian, P., Schick, F., Reischl, G., Pichler, B.J., 2014. Assessment of rodent

565 brain activity using combined [(15)O]H2O-PET and BOLD-fMRI. Neuroimage 89, 271-279.

566 https://doi.org/10.1016/j.neuroimage.2013.11.044

567 Yaniv, Z., Lowekamp, B.C., Johnson, H.J., Beare, R., 2018. SimplelTK Image-Analysis

568 Notebooks: a Collaborative Environment for Education and Reproducible Research. J Digit

569 Imaging 31, 290-303. https://doi.org/10.1007/s10278-017-0037-8

570 Yin, Y., Zhang, X., Williams, R., Wu, X., Anderson, D.D., Sonka, M., 2010.

571 LOGISMOS--layered optimal graph image segmentation of multiple objects and surfaces:

572 cartilage segmentation in the knee joint. IEEE Trans Med Imaging 29, 2023-2037. 
$573 \quad$ https://doi.org/10.1109/TMI.2010.2058861

574 Yu, C., Wang, J., Chen, Y., Huang, M., 2019. Transfer learning with dynamic adversarial

575 adaptation network. 2019 IEEE International Conference on Data Mining (ICDM). IEEE, pp.

576 778-786. https://doi.org/10.1109//CDM.2019.00088

577 Zerbi, V., Floriou-Servou, A., Markicevic, M., Vermeiren, Y., Sturman, O., Privitera, M., von

578 Ziegler, L., Ferrari, K.D., Weber, B., De Deyn, P.P., Wenderoth, N., Bohacek, J., 2019. Rapid

579 Reconfiguration of the Functional Connectome after Chemogenetic Locus Coeruleus

580 Activation. Neuron 103, 702-718 e705. https://doi.org/10.1016/j. neuron.2019.05.034

581 Zerbi, V., Grandjean, J., Rudin, M., Wenderoth, N., 2015. Mapping the mouse brain with

582 rs-fMRI: An optimized pipeline for functional network identification. Neuroimage 123, 11-21.

583 https://doi.org/10.1016/j.neuroimage.2015.07.090

584 Zhu, Y., Zhuang, F., Wang, J., Ke, G., Chen, J., Bian, J., Xiong, H., He, Q., 2021. Deep

585 Subdomain Adaptation Network for Image Classification. IEEE Transactions on Neural

$586 \quad$ Networks and Learning Systems 32, 1713-1722.

$587 \quad$ https://doi.org/10.1109/TNNLS.2020.2988928

588 